\title{
STATUS OF THE TAU-CHARM FACTORY PROJECT AND ASPECTS OF THE DETECTOR DESIGN $\ddagger$
}

\author{
Rafe H. Schindler \\ Stanford Linear Accelerator Center \\ Stanford University, Stanford, Califorriia 94309 USA
}

\begin{abstract}
This paper reviews the status of the Tau-Charm Factory Project being proposed for construction in Spain. The paper also reviews characteristics of the detector design, and the issues surrounding the present choices of technologies.
\end{abstract}

\section{THE TAU-CHARM PROJECT}

The Tau-Charm Project is an $\mathrm{e}^{+} \mathrm{e}^{-}$storage ring of high luminosity presently being proposed for construction in Spain. The machine has a design luminosity of $10^{33} \mathrm{~cm}^{-2} \mathrm{sec}^{-1}$ operating between 3 and $5 \mathrm{GeV}$ cms energy. The physics program of the machine is broad, encompassing both $\tau$ and charm production and decay studies near threshold, as well as studies of bound state charmonium production and decay. At design luminosity, the collider will produce close to $10^{8} \tau$ - pairs/yr, $10^{8}$ charmed mesons/yr., and $10^{10} \mathrm{~J} / \psi / \mathrm{yr}$.. As the tagging efficiency for charmed mesons and $\tau-10-20 \%$, samples exceeding $10^{7}$ reconstructed decays will become available, far exceeding any presently existing or proposed facility. The machine is being designed with monochrometer optics, allowing it to achieve cms energy spreads close to $100 \mathrm{KeV}$, and thereby allowing the production of $\mathrm{J} / \Psi$ to be increased to $2 \times 10^{11} / \mathrm{yr}$ for CP studies in the hyperon system. Beam polarization may also be routinely available.
A high luminosity collider operated at threshold for the pair production of charm and $\tau$, allnws us to take advantage of unique kinematic constraints to enhance the analyzing power of the detector, and allowing the search for rare processes at the $10^{-7}$ to $10^{-8}$ level. The issues of detector design are critical, to fully exploit the high luminosity; they are described in the balance of this paper.

\section{Tau-Charm Machine and Detector Interface}

The characteristics of the machine strongly influence the detector design. The machine is a double ring design, with electrostatic separators to minimize parasitic crossings of the beams to the IP region alone. The rings of $360 \mathrm{~m}$ circumference, each hold about 30 bunches, and have a crossing frequency of $25 \mathrm{MHz}$. The IR design has been evaluated by the CERN group. The vertical separators start at 3.5 meters from the IP (see Fig. 1 ), while the superconducting final focus quad. triplets penetrate the detector to $\pm 80 \mathrm{~cm}$ from the IP.

Talk. Presented at the 26th International Conference on High Energy Physics (ICHEP 92), Dallas, Texas, August 6-12, 1992

\footnotetext{
‡Work Supported By The U.S. Department of Energy Under Contract DE-AC03-76SF00515
} 
Aspects of The Tau-Charm Detector

The present design of the detector stresses a lung barrel $(|\cos \theta|<0.95$ containing a central drift chamber for tracking, followed by a $4 \mathrm{~m}$ long two iayer scintillating fiber time-of-flight system. The TOF itself is backed by a twofold longitudinally segmented CsI(Na) or CsI(Tl) crystal calorimeter, in a unique re-entrant geometry. The long barrel is chosen to avoid a significant endcap region. The hermeticity is completed by a forward silicon tracking system and calorimetry (luminosity monitor) comprised of $\mathrm{BaF} 2$ crystals. Unlike BFactory detector designs, the use of TOF for particle identification is adequate at TCF; scintillator, being a fully active medium, doesn't compromise calorimetry performance, as most other particle ID systems would. The hermeticity is completed by a fine grained muon range system which also acts as a veto for the energy carried by neucral hadrons, and as a flux return yoke. In this way, even neutrinos can be tagged by missing energy, an essential ingredient to the effectiveness of the detector for the study of rare decays and the tagging of $\tau$ - decays.

\section{Radiation Doses and Backgrounds}

The radiation background from the large circulating currents $(600 \mathrm{ma} /$ beam $)$ of the TCF rings has been analyzed for the ORSAY-optics, and assuming an optimal masking scheme. The luminosity lifetime of 2 hrs is dominated by beambeam bremstraulung assuming a $1 \mathrm{nT}$ vacuum can be maintained. Synchrotron radiation from the strong bending $(\rho \approx 12 \mathrm{~m}$ ) of the main the rings does not strike the deiector because of the vertical separation scheme, but it misi be arranged to avoid illumination of the opposing electrostatic separator plates. Synch. radiation from the vertical bends themselves and the final focus quads is very soft, and can largely be absorbed in the beryllium beampipe. The major sources of detector background is from electroproduced hadrons from beam gas interactions, from lost beam particles that are coulomb or bremstraulung scattered, and particles lost from the injected beam itself. The results from injection losses are least understood, and potentially the most serious as summarized in Table I. A model for injection frequency and loss rate is assumed

\section{THE TAU-CHARM FACTORY DETECTOR}

The basic features of the detector are described next. These requirements and potential solutions have largely been established through the work of the members of the US Tau-Charm Collaboration in R\&D carried out since 1989. The design of the tracking system for the TCF detector is motivated by the desire to accurately measure the endpoint spectrum of the decay of the $\tau$ into multihadrons. This will ultimately lead to the best resolution possible for measurement of a limit on the $v_{\tau}$-mass to about $1 \mathrm{MeV}$. To do this, the tracker must achieve a momentum (p) resolution of $0.35 \% \mathrm{p}$ and a multiple coulomb scattering term of about $0.35 \%$. At TCF energies, this is possible for a device having about 40 cylindrical planes of $\mathrm{Al}$ -guard/W-sense wires, over a radial extent of about $80 \mathrm{~cm}$, immersed in a $1 \mathrm{~T}$ axial magnetic field. We assume a low $\mathrm{Z}$ gas mixture such as $\mathrm{He}-\mathrm{CO}_{2}$ Isobutane is used, and knowledge of the IP position and sense and field wire multiple-scattering is included in the track fit. A single hit resolution of only about $150 \mu \mathrm{m}$ is required. The TCF detector does not need a vertex chamber, so the beampipe aperture can be maintained at about $r=5 \mathrm{~cm}$ through the IP, thereby reducing the broadband impedance of the machine. For the tracker, a first measurement in close proximity to the Be-pipe is important to eliminate the multiple scattering contribution to angular error in the long track extrapolation to the IP. The primary difficulty in TCF will be the $3.6 \mathrm{~m}$ long wires required to meet the solid angleconstraints of the long barrel design. The electronics and endplate materials must also be minimized, or relocated, to avoid damage to the calorimetry performance for $0.95>|\cos \theta|>0.90$.

Following the tracking system, $\pi, \mathrm{K}, \mathrm{p}$ particle ID is accomslished most directly by a system of about 96 azimuthally divided fiber TOF counters. Two radial laytrs, each $5 \mathrm{~cm}$ thick of $1 \mathrm{~mm}$ fiber, and readout on both ends with XP2020 


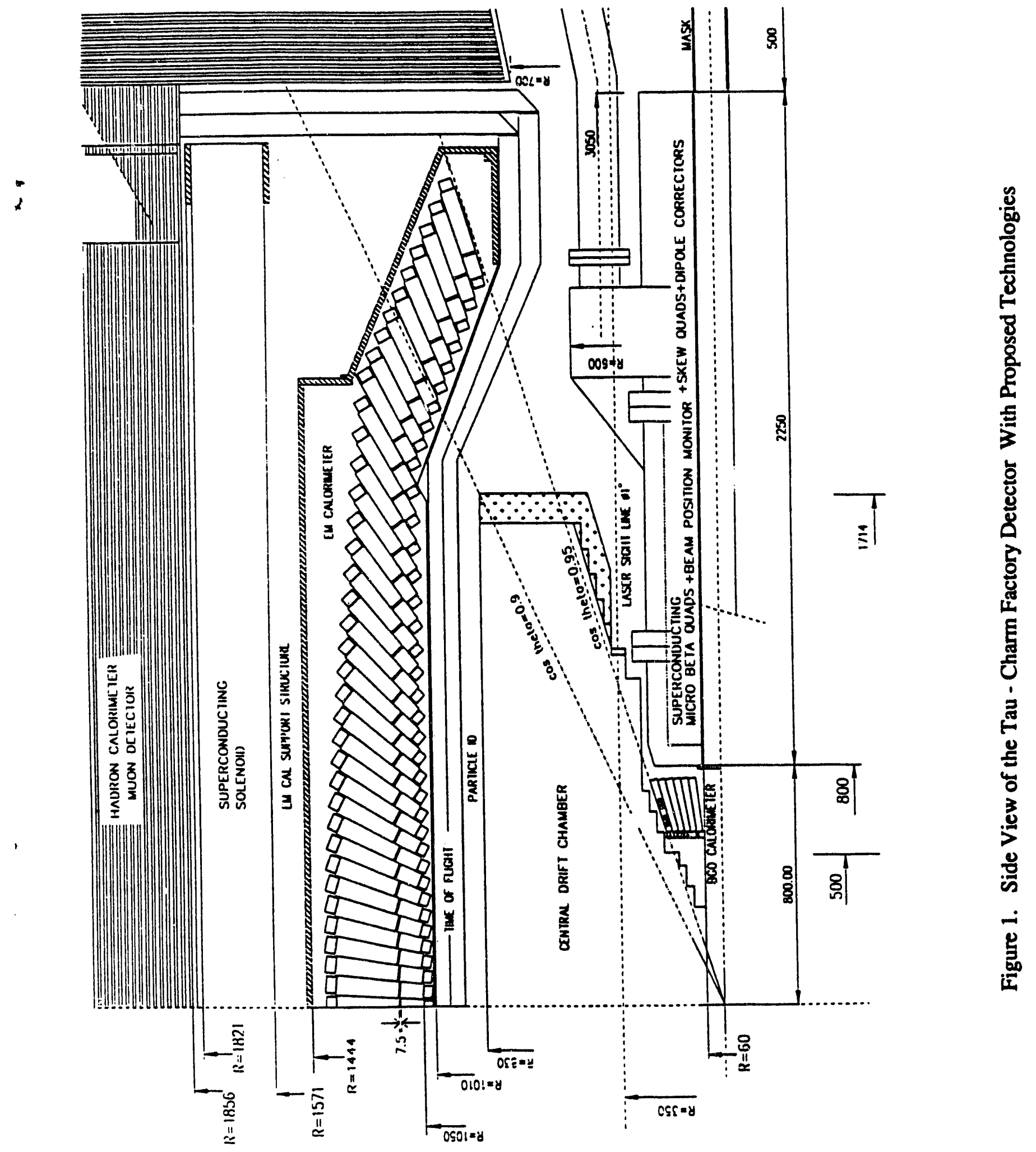


equivalent photomultiplier tubes, are required to achieve a time resolution of $120 \mathrm{ps}$ over the 1 meter flight path. This is required primarily to give adequate background rejection $(>3 \sigma)$ in the measl:rement of $D^{0} \bar{D}^{0}$ mixing, where $\pi$ and $K$ momenta approach $1 \mathrm{GeV}$. Fiber counters are optimal because of their greater attenuation length appropriate to the long barrel design. They can also be easily tailored to the re-entrant barrel design. In this present design, they match up to clear fiber highways, which then penetrate radially outward at the end-door breaks. The PMTs are then located outside the magnetic field. As indicated earlier, the TOF system will not degrade the calorimeter, as it itself is a fully active absorber medium.

The CsI calorimeter is one of the most important features of the TCF detector. CLEO-II was the first experiment to recognize the power of bringing high resolution and high detection efficiency calorimetry together with a large solid angle tracking system, for the precision studies of heavy quark decays. In TCF, where the physics is even more exclusive, and kinematic fitting is effective, the concept of having both photons and charged tracks measured with similar precision, has been demonstrated by our Monte Carlo studies to be unusually powerful in the study of rare processes where not just statistics but also backgrounds and systematics are important. CsI is an ideal crystal, producing enough light to make possible the detection of $10 \mathrm{MeV}$ photons with full efficiency while retaining a roughly $2 \% / \mathrm{E}^{0.25}$ resolution function. $R \& D$ for a CsI calorimeter is being carried out by the US Collaboration. The work encompasses mechanical design and fabrication of a carbon fiber structure, automated crystal polishing and tuning techniques, radiation damage characterization of crystals, the development of new wavelength shifters and photodiodes, the development of application specific integrated circuits for the front end electronics, and studies optical calibration of the crystals.

In our present design, both $\mathrm{CsI}(\mathrm{Tl})$ and $\mathrm{CsI}(\mathrm{Na})$ crystals of $16 \mathrm{rl}$ depth, read out with wavelength shifters and PIN photodiodes are being investigated (see Fig. 2). The primary advantage of CsI crystal is its large light output (about $50 \mathrm{~K}$
Table I. Radiation Dose From Backgrounds

\begin{tabular}{lll}
\hline \hline Section & $\begin{array}{l}\text { Background } \\
\text { Sources }\end{array}$ & $\begin{array}{l}\text { Doses } \\
\text { (Rad/Yr) }\end{array}$ \\
\hline BGO & Beam-Gas & 22 \\
& Lost Particles & 168 \\
& $0^{0}$ Rad. Bhabha & 0.03 \\
& Bhabhas & 5 \\
& Injection & 3400 \\
CDC & Non-Injection & $1 \times 10^{-2} \mathrm{C} / \mathrm{cm} / \mathrm{yr}$ \\
& Injection & $0.2 \mathrm{C} / \mathrm{cm} / \mathrm{yr}$ \\
TOF & Lost Particles & 1.1 \\
& 0 & 0.4 \\
& Injection & 22 \\
CsI & Beam-Gas & $10^{-4}$ \\
& Lost Particles & 0.23 \\
& $0^{0}$ Rad. Bhabha & 0.1 \\
& Injection & 4.6 \\
\hline \hline
\end{tabular}

photons/MeV), however the drawback is its long time constant $(1 \mu \mathrm{sec}, 0.5 \mu \mathrm{sec}$ for $\mathrm{Tl}$ and $\mathrm{Na}$ respectively). The consequence of this is the need to do coarse timing ( $\sim 40 \mathrm{~ns})$ on the output, to reduce machine background pileup. Energy of showers must be uniquely assigned to beam crossings.

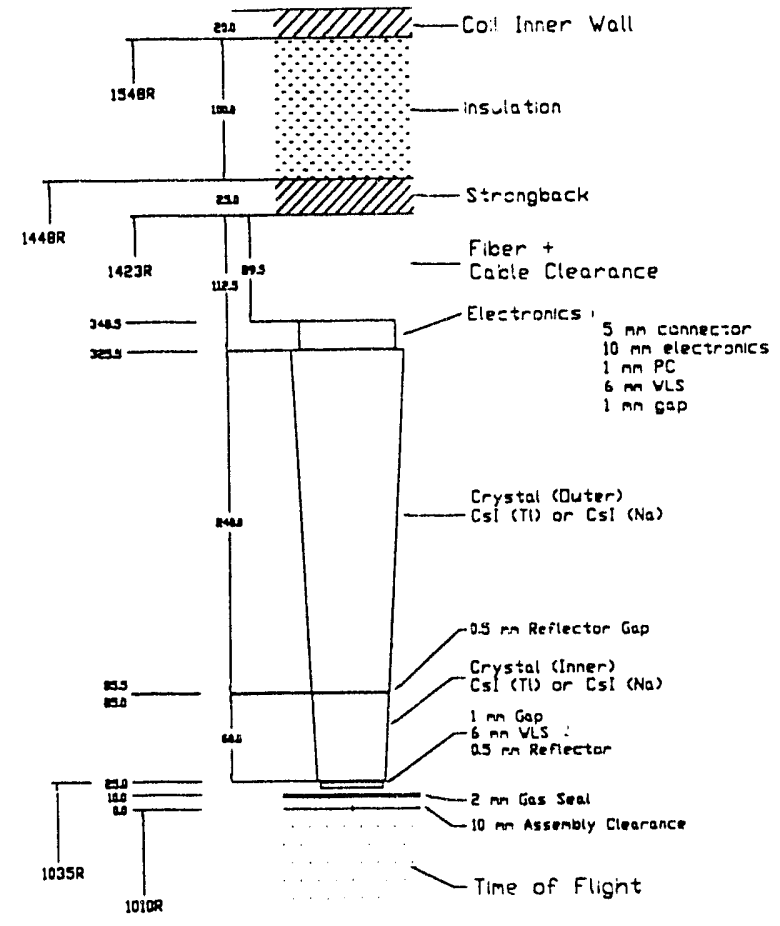

Figure 2. An Option For CsI Crystal Geometry 
This will leave only the problem of albedo showers within the time window of $40 \mathrm{~ns}$ of a beam crossing.

To further address this remaining problem of in-time backgrounds we are investigatiing the use of increasing the longitudinal and transverse segmentation and readout of crystals, as illustrated in Fig. 2. These will provide additional information on shower shape, origin and direction. An extensive beam test program containing $22 \mathrm{~K}$ cc of CsI, arranged with 132 readout channels was carried out in August 1992 at TRIUMF to study the strengths of these duel split crystal geometries and front/back wavelength shifter readout. Results will be reported in the near future.

The calorimetric solid angle is closed by a $\mathrm{BaF}_{2}$ array in the very forward direction, that is mounted on the face of the final quads. This array doubles as the luminosity monitor, being supplemented by several planes of a large area silicon pad tracking system. This strip and pad system will be pre-processed by an onboard VLSI chip. Tests by members of the German TCF Collaboration have already been carried out at CERN on prototypes of the silicon tracking elements, and a detailed design for the crystal device is now under study by those groups.

Radially outward, following the CsI calorimeter, is the thin superconducting $1 \mathrm{~T}$ coil. While it is outside the calorimetry, we still wish to do range measurements for muon identification, so the coil, its terminations and its cryostat must be minimized. A 0.25 absorption length device is acceptable. The muon system that follows is extremely fine grained, having steel plates of 1.5 to $2 \mathrm{~cm}$ thickness. Typical range differences at 500 $\mathrm{MeV} / \mathrm{c}$ between pions and muons are only 2 to 3 such gaps. Because of the low occupancy, we envision a large cell drift chamber between absorber layers. The requirement on the overall efficiency per layer of the device is extremely tight; it must achieve greater than $95 \%$ (including geometric occlusions) efficiency since the muon range system is also necessary as a veto for energy from neutral hadrons $\left(\mathrm{K}^{0} \mathrm{~L}\right)$, and will impact rare decay studies if it is not sufficiently hermetic and efficient.

\section{Electronics and Data Acquisition}

Lastly, I will comment on the electronics and data acquisition. The drift chamber has about $5 \mathrm{~K}$ wires, the calorimeter about $20 \mathrm{~K}$ elements, forward calorimeters about $1 \mathrm{~K}$ elements, the TOF about $1 \mathrm{~K}$ ADC/TDC's, and the muon system about $20 \mathrm{~K}$ wires. While this detector is then very comparable to present SLC and LEP detectors, perhaps unique to the TCF detector is the large dynamic range required in the trigger. The trigger must span from $100 \mathrm{~Hz}$ to about $5 \mathrm{KHz}$ of true physics events. The purity of the trigger is expected to exceed $50 \%$ namely a background rate under $100 \mathrm{~Hz}$ from beam related sources is anticipated. A typical event should be processed in the front-end electronics to contain less than about 10 Kbytes of data. We envision extensive onboard detector signal processing and pattern recognition of raw data, directly into either digital or analog pipelines, thereby allowing a deadtimeless trigger to be formed. The final stages of the trigger would process the event more fully, applying geometric and time-varying constants, and allowing a very high level topological discrimination to take place, before writing events to a storage device. While such processing is not required for the running at energies suitable for $\tau$ and charm physics studies, this feature is essential if the machine is run with monochrometer optics on the $J / \psi$, where the event rate will increase by 1 order of magnitude.

\section{ACKNOWLEDGMENTS}

I would like to thank Juan Antonio Rubio for his encouragement and his single-handed leadership in what is now an international effort to build a Tau-Charm Factory Laboratory in Spain. I would also like to acknowledge the many other members of the U.S .Tau-Charm Collaboration for their strong support in the R\&D program for the calorimetry, and their intellectual input into the overall TCF detector design and physics simulation effort. 

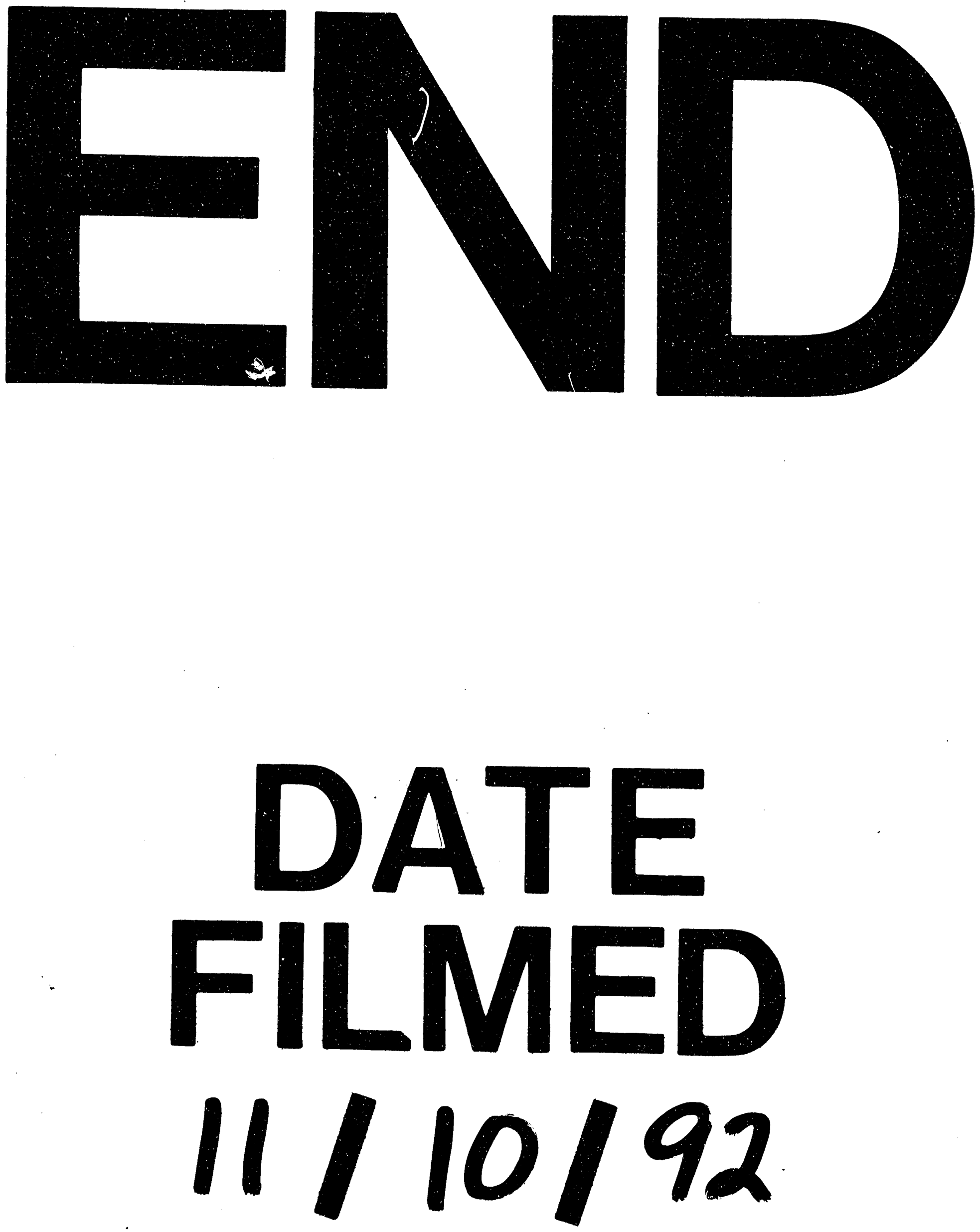
